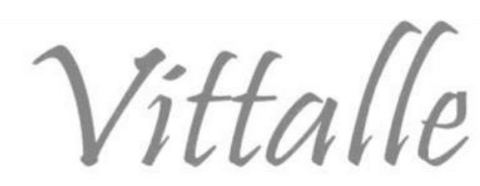

\title{
Implementação da Visita Familiar Ampliada na Unidade de Terapia Intensiva Adulto de um Hospital Universitário
}

\author{
Letícia Macedo Gabarra*, Camila Louise Baena Ferreira, Paula Adriane Lombardi \\ Hospital Universitário Professor Polydoro Ernani de São Thiago, Universidade Federal de Santa Catarina \\ (HU/UFSC/EBSERH), Florianópolis, SC, Brasil
}

\begin{abstract}
Histórico do Artigo
Recebido em:

01/12/2019

Aceito em:

$14 / 03 / 2020$

Palavras-chave:

Unidades de Terapia

Intensiva; visitas a

pacientes; equipe de

assistência ao

paciente; psicologia

Keywords:

Intensive Care Units; visitors to patients; patient care team; psychology

RESUMO

A admissão de pacientes em Unidades de Terapia Intensiva (UTI's) gera repercussões emocionais no indivíduo e familiares. Estas unidades remetem à gravidade da condição clínica e geralmente, possuem política restritiva de visitação. O presente artigo apresenta como objetivo descrever o processo de implementação da visita familiar ampliada - até 12 horas ininterruptas - na Unidade de Terapia Intensiva Adulto de um Hospital Universitário, sob a perspectiva da equipe de Psicologia do setor. É apresentado, conforme estabelecido junto com a equipe multiprofissional, os critérios de seleção das famílias e aspectos a serem avaliados antes de efetivar a inclusão na visita ampliada. A implementação da visita ampliada tem se mostrado viável, com boa aceitação dos familiares, pacientes e equipe. Apresenta-se como resultado, o alívio da ansiedade dos familiares e o aumento da segurança e confiança na equipe multiprofissional. Os pacientes consideram positiva a experiência da visita ampliada e apresentam maior benefício especialmente quando apresentam sintomas de ansiedade, humor deprimido, agitação, delirium conforme observado pela equipe assistencial.

The Implementation of Extended Family Visit at the Adult Intensive Care Unit of a University Hospital

\section{ABSTRACT}

The admission of patients to Intensive Care Units (ICUs) generates emotional repercussions on the individual and family members. These units refer to the severity of the clinical condition and generally have a restrictive visitation policy. This article describe the process of the implementation extended family visit - up to 12 uninterrupted hours - in the Adult Intensive Care Unit of a University Hospital, from the perspective of the sector's Psychology team. As established with the multiprofessional team, the criteria for selecting families and aspects to be evaluated before inclusion in the extended family visit. The implementation of the extended visit has been shown to be viable, with good acceptance by family members, patients and professionals. As a result, relief from family anxiety and increased security and confidence in the multiprofessional team are presented. Patients consider the extended visit experience to be positive and are most beneficial especially when they have symptoms of anxiety, depressed mood, agitation, delirium as noted by the professionals.
\end{abstract}

\section{Introdução}

As Unidades de Terapia Intensiva (UTI's) são unidades hospitalares voltadas ao atendimento de pacientes graves ou com risco de gravidade clínica, que necessitam de assistência profissional ininterruptas, com acesso a recursos humanos especializados e tecnologias destinadas a diagnósticos e terapêuticas (1). O processo de adoecimento é compreendido como um estressor importante na vida do indivíduo acarretando repercussões emocionais tanto para o próprio paciente como para sua família. Quando esse processo requer internação em UTI, entende-se que o impacto pode ser exacerbado pois esta unidade está associada à gravidade da condição de saúde do paciente que

\footnotetext{
* Autor correspondente: leticiagabarra@gmail.com (Gabarra L.M.)
} 
requer cuidados intensivos da equipe multiprofissional e, inevitavelmente remete à iminência de morte (2-4).

Com o desenvolvimento tecnológico e o deslocamento do centro de cuidado da comunidade para o ambiente hospitalar, a rede social do indivíduo foi destituída de seu papel de cuidado. É possível observar na planta física dos hospitais e nas rotinas hospitalares, a ausência de espaço para acompanhantes e visitantes (5). Tal situação torna-se bastante evidente nas UTI's, pois geralmente possuem horário de visita restrito $(2,6)$ de até 60 minutos no máximo $(6)$, e não permitem acompanhante ao paciente como as demais unidades do Hospital.

Conforme a Resolução que estabelece os requisitos mínimos para as UTI's em território nacional, todos os profissionais da unidade devem assegurar o incentivo à participação da família na atenção ao paciente, quando possível (7). Ressalta-se que, entre as principais preocupações dos familiares de pacientes internados em UTI, encontra-se o período reduzido de tempo em que permanecem junto ao paciente durantes as visitas (3) e aspectos relacionados à comunicação com a equipe assistencial para obter maiores informações sobre o paciente e o tratamento oferecido $(8,9)$. A presença dos familiares é importante para ajudar na identificação de dados do contexto de vida do paciente e suas necessidades, favorecendo assim a qualidade na assistência prestada pelos profissionais; assim como a manutenção do vínculo afetivo visando proporcionar uma melhor adaptação do paciente na unidade $(10,11)$.

Historicamente, a justificativa para a política de restrição de visitas em UTI está relacionada à crença no aumento da taxa de infecção hospitalar e o prejuízo no trabalho da equipe assistencial (12-15). Entretanto, autores demonstram que não foram encontradas evidências para sugerir que os visitantes apresentem um risco direto de infecção para os pacientes (16-20). Apontam que a visita dos familiares em tempo ampliado fortalece o vínculo com a equipe assistencial, favorecendo a saúde emocional dos pacientes e familiares, os quais sentem-se mais seguros quanto ao tratamento disponibilizado $(2,21)$.

No Brasil, a implementação da visita ampliada é um fato em diversos hospitais em todo o país $(19,22)$. A presença do familiar em tempo estendido de visita, possibilita benefícios tanto para os pacientes, como para os familiares. Sendo assim, nos hospitais nos quais isso ocorre, oferecem às famílias elegíveis para visita ampliada a possibilidade de até duas pessoas por paciente permanecerem junto ao mesmo, por no máximo doze horas por dia. Os familiares recebem orientações com foco em boas práticas de visitação e aspectos da segurança do paciente, medidas de prevenção de delirium e de transmissão de infecções $(19,20,22)$.

Espera-se que a inserção da família dentro da UTI ao lado de seu ente que se encontra hospitalizado, fragilizado fisicamente e emocionalmente devido o processo de adoecimento, possa proporcionar um atendimento mais humanizado, centrado nas necessidades do paciente e de sua família (23). A presença dos familiares na UTI por um período ampliado pode minimizar os desconfortos vivenciados pelas famílias e pacientes, desde que associada ao acolhimento e comunicação efetiva da equipe de saúde (10).

Pesquisa realizada com médicos e enfermeiros demonstrou que a maioria $(58,1 \%)$ opinaram que a política de visitação em UTI's deveria ser menos restritiva em relação ao tempo e número de visitantes permitidos e apenas 3,2\% sugeriram que a política de visitação deveria ser mais restritiva (6). Entretanto, outra pesquisa apresentou que $59,4 \%$ dos profissionais acreditam que a visita ampliada prejudica a organização do atendimento ao paciente (14); sendo que a família demonstra uma perspectiva mais positiva em relação à visita ampliada que a equipe assistencial em diversos aspectos (11, 
24). Observa-se que o tempo de experiência profissional, número de profissionais na unidade e número de turnos noturnos trabalhados foram fatores preditivos das atitudes e crenças dos enfermeiros em relação às crenças sobre a visita da família (25).

Neste sentido, o presente artigo pretende descrever o processo de implementação da Visita Familiar Ampliada na Unidade de Terapia Intensiva Adulto de um Hospital Universitário sob a perspectiva da equipe de Psicologia do setor.

\section{Metodologia}

Trata-se de um relato de experiência sobre as vivências de profissionais da Psicologia durante o processo de implementação da visita familiar ampliada na Unidade de Terapia Intensiva Adulto (UTI-A) do Hospital Universitário Professor Polydoro Ernani de São Thiago - vinculado à Universidade Federal de Santa Catarina (HU/UFSC). A unidade se caracteriza como de médio porte, com 12 leitos ativos destinados aos pacientes a partir dos 15 anos em estado clínico grave ou pós-operatório imediato. A equipe multiprofissional é composta por médicos, enfermeiros, técnicos de enfermagem, psicólogos, assistentes sociais, nutricionistas, fisioterapeutas, fonoaudiólogos, farmacêuticos, dentistas, nutrólogos e terapeuta ocupacional; e os residentes das respectivas áreas.

Destaca-se que o perfil dos pacientes atendidos são jovens adultos e idosos, com adoecimento crônico agudizado com necessidade de cuidados intensivos, e em alguns casos também paliativos. A Instituição é referência em toxicologia com atendimento a situações de intoxicações exógenas, incluindo tentativas de suicídio; referência em hepatologia com a realização de transplantes hepático; e referência em cirurgia vascular. Nessa instituição não há atendimento de trauma (neurocirurgia e ortopedia), nem tampouco cirurgias cardíacas.

O referido hospital integra desde 2017 o projeto colaborativo "Melhorando a Segurança do Paciente em Larga Escala no Brasil", juntamente com outros 119 hospitais em todo o país. O projeto foi idealizado pelo Ministério da Saúde, através do Programa de Desenvolvimento Institucional do Sistema Único de Saúde (PROADISUS). Conta com a parceria de cinco hospitais de excelência - Albert Einstein, Instituto do Coração, Oswaldo Cruz, Moinhos de Vento e Sírio-Libanês, que trabalham de forma colaborativa e utilizam a metodologia e apoio técnico do Institute for Healthcare Improvement - IHI. Cabe ressaltar que, o Hospital Moinhos de Ventos (Porto Alegre/RS) é o atual responsável pela supervisão do Projeto no HU/UFSC (26).

O objetivo é melhorar a segurança do paciente com a implantação de práticas de prevenção de infecções relacionadas ao uso de ventilação mecânica, uso de cateteres venosos e de sondas vesicais. Dentre as ações previstas no projeto colaborativo, encontra-se a implementação da Visita Familiar Ampliada na UTI-A (26). Para a efetivação do projeto, os hospitais são capacitados para implantar as medidas de prevenção por meio de Ciclos de Melhorias, utilizando o ciclo PDSA (plan - do - study act), para o processo ser sustentado e praticado por toda equipe. O projeto proporciona sessões de aprendizagem presenciais (SAP) e virtuais (SAV) para a troca de experiências e acompanhamento dos resultados.

No presente artigo são descritas as experiências profissionais das Psicólogas pertencentes ao Serviço de Psicologia do hospital e referências para a UTI-A, assim como da Psicóloga Residente imersa neste setor, pertencente à Residência Integrada Multiprofissional em Saúde com ênfase em Alta Complexidade. Para tanto, utilizou-se da vivência das profissionais em atendimentos psicológico aos pacientes e familiares da UTI, participações em reuniões da equipe multiprofissional, discussões e reuniões com 
a equipe do HU/UFSC e Hospital Moinhos de Vento pertencentes ao projeto "Melhorando a Segurança do Paciente em Larga Escala no Brasil"; no período de um ano, entre dezembro de 2018 a dezembro de 2019.

A experiência das autoras, juntamente com a participação das equipes multiprofissionais engajadas na implementação do projeto e dos pacientes e familiares que experienciaram esse processo, foram essenciais para o presente relato de experiência. Tendo em vista que o ponto de partida foi o cotidiano do trabalho das autoras, assim como as observações e reflexões que surgiram com a mudança no processo de trabalho e funcionamento da unidade já consolidada ao longo do tempo.

\section{Resultados e Discussão}

A experiência relatada neste artigo busca apresentar como a equipe assistencial da UTI-A do HU/UFSC organizou a visita ampliada conforme os recursos humanos e estruturais que possui. Ressalta-se que a política de visitação deve ser organizada em cada unidade conforme as particularidades do serviço. Afinal, pensar a visita ampliada envolve ampliar o tempo e número de visitantes amparado nos aspectos estruturais, administrativos e socioculturais que influenciam na manutenção de determinadas práticas de visitação restritivas (10). Observa-se, entretanto, que as necessidades dos familiares de pacientes internados em UTI apresentam pouca relação com aspectos físicos do ambiente; valorizando as questões relacionadas à comunicação e informação acerca do paciente $(8,9)$.

A UTI-A do HU/UFSC disponibiliza três horários diários de visitas para os pacientes entre os períodos matutino, vespertino e noturno; totalizando 02 horas e 30 minutos no total. No final de semana, os pacientes são assistidos por equipe em turno de plantão (12 horas) e o tempo de visitação é reduzido para 2 horas no total, nos períodos vespertino e noturno. Anteriormente à implementação da visita ampliada, ocorria a flexibilização de visitas apenas em situações específicas consideradas exceções, como por exemplo, em processos de terminalidade da vida, em casos de menores de idade e de pessoas com deficiência.

A primeira etapa para implementação da visita ampliada na UTI-A do HU/UFSC iniciou em 2018 através de reuniões internas da equipe multiprofissional da Unidade; e reuniões com a participação da equipe do Hospital Moinhos de Ventos, nas quais fizeram sugestões de como viabilizar tal prática. Os temas contemplados nesses encontros foram: horário da visita ampliada; quantidade de visitantes em tal modalidade; critérios de seleção; orientações, infraestrutura e alimentação a serem fornecidas aos visitantes; monitoramento da visita. $\mathrm{O}$ envolvimento da equipe multiprofissional foi relevante para compreender a crença acerca da visita ampliada, pois conforme demonstrado na literatura, a equipe assistencial pode apresentar resistência à visita ampliada por acreditar que irá acarretar prejuízos na organização do trabalho na unidade $(14,15,27)$.

Desse modo, a estruturação das visitas ampliadas levou em consideração a opinião dos profissionais do setor visando elaborar estratégias para manter a organização da rotina na unidade e não sobrecarregar o trabalho dos servidores. Assim, após diversas reuniões com a equipe, realizações de PDSA com experiência de visita ampliada e estudos a partir das experiências em outras UTI's, ficou estabelecido os seguintes critérios para o benefício da visita ampliada: a) pacientes menores de 18 anos ou pessoas com deficiência de qualquer idade, independente da condição clínica, tem direito a acompanhante - conforme estabelecido pelo Estatuto da Criança e do Adolescente ECA (28) e Estatuto da Pessoa com Deficiência (29); b) pacientes maiores de 18 anos, fora da ventilação mecânica, acordado e contactuante; c) a critério clínico: pacientes em 
isolamento e outras situações que serão avaliadas pontualmente pelo corpo clínico. Ressalta-se que a visita ampliada pode ser concedida apenas aos familiares maiores de 18 anos (30).

Observa-se que a visita ampliada foi priorizada aos pacientes em condições de interagir com a família, entretanto há a possibilidade desta modalidade de visita também aos pacientes em uso de ventilação mecânica e/ou não contactuantes; conforme avaliação da equipe assistencial acerca do benefício para os pacientes e familiares. Assim, diversos profissionais da equipe assistencial podem detectar pacientes que apresentam os critérios para receber visita ampliada e encaminhar aos profissionais da Psicologia e Serviço Social, responsáveis pela avaliação dos familiares e orientações conforme o fluxograma apresentado na Figura 1.

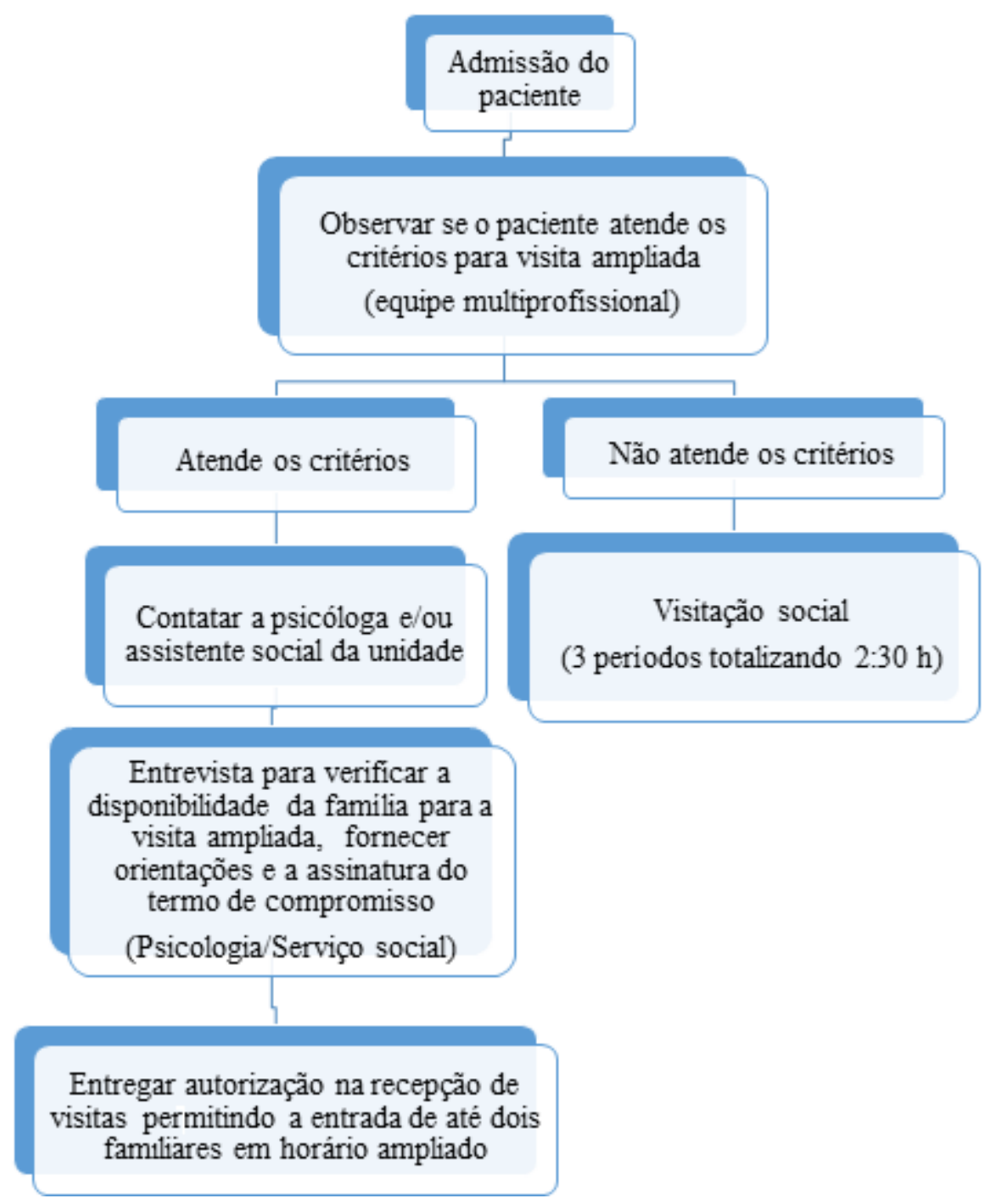

Figura 1 - Fluxograma da visita ampliada na UTI-A HU/UFSC.

$\mathrm{Na}$ avaliação realizada pelo Serviço de Psicologia busca-se verificar a disponibilidade da família, compreensão da organização e rotina da unidade, postura colaborativa frente às orientações a serem seguidas; assim como os aspectos emocionais, atentando-se para o real benefício para o paciente e/ou para sua família. Eventualmente, determinados familiares podem apresentar intensa ansiedade e contribuir para sintomas de agitação no paciente; assim, não estariam elegíveis para a visita ampliada nesse contexto. Observase que alguns familiares possuem comportamento evitativo durante as visitas sociais, 
permanecendo poucos minutos junto ao paciente no leito; apresentam-se angustiados e fragilizados emocionalmente diante do contato com o paciente em uso de dispositivos invasivos, com alteração significativa da imagem corporal e risco de falecimento.

Entretanto, existem as famílias que expressam alívio dos sintomas de ansiedade ao permanecerem ao lado do seu familiar internado na UTI na visita ampliada, corroborando com a literatura $(11,20,25)$. A presença dos familiares junto ao leito, apesar de apresentar aspectos geradores de desconforto ao visualizar o paciente requerendo cuidados numa condição diferente do habitual, proporciona segurança frente ao tratamento que vem sendo disponibilizado ao mesmo. Os familiares sentem-se satisfeitos com a possibilidade de realizar companhia para o paciente durante esse momento de fragilidade física e emocional (31).

As orientações fornecidas às famílias que permanecerão em visita ampliada acontecem através do Termo de Compromisso, no qual são identificados dois familiares que podem revezar a permanência na UTI por até 12 horas (entre às 09:00 e 21:00). Desse modo, apenas um familiar pode permanecer junto ao leito do paciente; e é fornecida alimentação no refeitório do Hospital ao familiar. O psicólogo realiza orientações sobre a rotina da UTI e práticas de boa visitação, com foco em práticas de segurança do paciente. Explica-se aos familiares, entre outras questões, sobre a possibilidade da equipe solicitar que se retirem do leito e aguardem na sala de espera para a realização de procedimentos invasivos no paciente.

Após o estabelecimento dos critérios e do fluxograma apresentado, a visita ampliada foi efetivada no ano de 2019. A maioria das famílias selecionadas demonstraram interesse e disponibilidade em participar da visita ampliada. O fator impeditivo para maior adesão das famílias tem sido a necessidade de manter sua rotina laboral, visto a impossibilidade de abono de faltas e/ou dispensa do trabalho para acompanhamento de familiar na UTI. Entretanto, mesmo os familiares impossibilitados de permanecer 12 horas afirmam benefícios com a visita ampliada, visto a flexibilidade de horário, sem a obrigatoriedade de permanência em todo tempo.

Observa-se que algumas famílias, ao serem comunicadas que o paciente necessitará de cuidados pós operatórios na UTI, solicitam sua inclusão na visita ampliada antes mesmo da admissão do paciente na unidade; relatam possuir conhecimento acerca da visita ampliada através de outros pacientes e familiares que vivenciaram a experiência. Enquanto, outras famílias com paciente em pós operatório na UTI demonstram interesse em realizar visitas sociais, com horário mais restritivo, pois esse se torna o tempo para descansar e posteriormente continuar o acompanhamento ao paciente na enfermaria.

As famílias em visita ampliada identificam o benefício de obter informações para além do boletim médico, através do contato contínuo com a equipe multiprofissional. Demonstram assim, possuir melhor compreensão da condição clínica e prognóstico do paciente diante dos contatos pontuais com a equipe médica e de enfermagem/técnicos que constantemente entram no leito para realizar os cuidados; assim como demais membros da equipe multiprofissional. As famílias também relatam satisfação pela oportunidade de conhecer os diversos profissionais que realizam os cuidados ao paciente; pois devido ao horário restritivo da visitação social, nesta se torna mais difícil à família conhecer todos os profissionais.

Constata-se ainda, que não apenas os familiares mais próximos do paciente, fisicamente e/ou afetivamente, demonstram interesse e disponibilidade para a visita ampliada. A experiência de adoecimento e hospitalização é capaz de modificar o movimento em que os familiares se encontram, pode provocar uma "força centrípeta", ou seja, a família se volta para a doença e/ou para o doente mesmo quando se encontra num período de afastamento (32). Desse modo, a visita ampliada torna-se um importante recurso para proporcionar o 
reestabelecimento dos vínculos afetivos familiares.

O Hospital Universitário deste relato recebe pacientes de outros municípios do Estado de Santa Catarina, assim como familiares procedentes de outras regiões. Desse modo, o Serviço Social possibilita que a família disponha de acomodação em casa de apoio social. Tais familiares, especialmente, demonstram satisfação com a visita ampliada já que muitas vezes se encontram sozinhos na cidade de Florianópolis e sentem-se angustiados de ter contato com o paciente apenas durante 2 horas e 30 minutos diários como realizado na visita social.

A possibilidade de acompanhante 24 horas tem sido autorizada em alguns casos, conforme a condição clínica ou benefício psicossocial para o paciente e familiar. Nesse sentido, a visita ampliada se estende para acompanhante 24 horas, em especial nos casos de puérperas com perda gestacional, pacientes em pós operatórios de transplante hepático com várias intervenções e pacientes jovens adultos (entre 18 e 21 anos, aproximadamente). Observa-se nessas situações que os pacientes se sentem mais seguros no período noturno, expressam conseguir descansar melhor e apresentam melhor adaptação à internação na unidade. Há também a situação de familiares, angustiados diante do quadro clínico do paciente, que recusam a se distanciar da UTI e utilizam a sala de espera para dormir conforme observado na experiência de trabalho cotidiano e na literatura (33). Tal situação afirma ainda mais a necessidade de efetivar a visita ampliada e até mesmo permitir o acompanhamento 24 horas, em casos específicos.

O fluxo de entrada e saída dos familiares em visita ampliada na UTI tem funcionado bem, com a participação efetiva dos funcionários da recepção do HU, assim como da equipe da UTI. A gestão hospitalar tem oferecido o suporte necessário para concretização das ações propostas para a visita ampliada, como o fornecimento das alimentações aos acompanhantes e através da estrutura física instalada. A UTI-A do HU/UFSC possui sala de espera e sala de reuniões, permitindo um ambiente mais acolhedor e reservado entre a família e equipe quando se torna necessário; enquanto a literatura demonstra que um número significativo de UTI's brasileiras não dispõe de sala de espera e de reunião (6).

Durante o ano de implementação, observou-se a maior participação da equipe na indicação de famílias para a visita ampliada; assim como a solicitação de presença de familiares para os casos de pacientes ansiosos, agitados, apáticos, deprimidos, confusos e em delirium. Percebe-se a integração da equipe no acolhimento ao familiar, e na adequação dos procedimentos e rotinas diante da presença dos visitantes.

\section{Considerações Finais}

A implementação da visita ampliada na UTI-A do HU/UFSC é recente, visto que sua efetivação ocorreu no ano de 2019; e tem se mostrado um projeto viável conforme a organização aqui relatada. A equipe assistencial da UTI demonstrou boa aceitação e frequentemente contatam o Serviço de Psicologia após identificar pacientes que atendem aos critérios para visita ampliada. As famílias, por sua vez, demonstraram alívio dos sintomas de ansiedade, e o aumento da segurança e confiança na equipe proporcionados pela proximidade de contato e possibilidade de obter informações recorrentes acerca do paciente. Benefícios são relatados também por parte dos pacientes, que demonstram satisfação com a companhia de familiares; e necessitam de tal contato especialmente nos casos que apresentam sintomas de ansiedade, humor deprimido e/ou agitação conforme observado pela equipe multiprofissional.

Constata-se que a visita ampliada se tornou de conhecimento da instituição como um 
todo, diante da comunicação entre os profissionais das diferentes unidades assim como o relato dos familiares e pacientes que vivenciaram tal experiência. Desse modo, observa-se que a demanda por visita ampliada tende a aumentar na unidade e faz-se necessário pesquisas acerca do impacto emocional para os pacientes e famílias que vivenciaram a visita ampliada, assim como para os profissionais da UTI que se encontram inseridos nesse ambiente de recente transformação.

\section{Referências}

1. Ministério da Saúde (Brasil). Portaria $n^{\circ} .3432$, de 12 de agosto de 1998. Estabelece critérios de classificação para as Unidades de Tratamento Intensivo - UTI. Diário Oficial da União 12 ago 1998; Seção 1.

2. Nunes MEP, Gabarra LM. Percepção de familiares sobre visitas a pacientes e regras em unidade de terapia intensiva. Arq. Ciênc. Saúde 2017; 24(3): 84-88.

3. Lima F de A, Amazonas MCL de A, Barreto CLBT, Menezes WN de. Sons and daughters with a parent hospitalized in an Intensive Care Unit. Estud. psicol. (Campinas) 2013; 30(2): 199-209.

4. Hunter JD, Goddard C, Rothwell M, Ketharaju S, Cooper H. A survey of intensive care unit visiting policies in the United Kingdom. Anaesthesia 2010; 65(11):1101-1105

5. Ministério da Saúde. Secretaria de Atenção à Saúde. Núcleo Técnico da Política Nacional de Humanização. HumanizaSUS: visita aberta e direito ao acompanhante. Brasília: MS, 2007 (MS. Textos Básicos de Saúde).

6. Ramos FJ da S, Fumis RRL, Azevedo LCP de, Schettino G. Políticas de visitação em unidades de terapia intensiva no Brasil: um levantamento multicêntrico. Ver Bras Ter Intensiva 2014; 26(4): 339-346.

7. Agência Nacional de Vigilância Sanitária (Brasil). Resolução n.7, de 24 de fevereiro de 2010. Dispõe sobre os requisitos mínimos para funcionamento de Unidades de Terapia Intensiva e dá outras providências. Diário Oficial da União 24 fev 2010; Seção 1.

8. Puggina AC, Ienne A, Carbonari KFBS da F, Parejo LS, Sapatini TF, Silva MJP. Percepção da comunicação, satisfação e necessidades dos familiares em Unidade de Terapia Intensiva. Esc Anna Nery 2014; 18(2):277-283.

9. Batista VC, Monteschio LVC, Godoy FJ de, Goés HL de F, Matsuda LM, Marcon SS. Necessidades de familiares de pacientes internados em Unidade de Terapia Intensiva. Rev Fund Care Online 2019; 11(n.esp):540-546.

10. Eugênio CS, Filho MCB, Souza EM de. Visita aberta em UTI adulto: utopia ou realidade? Rev Enferm UFSM 2017; 7(3): 539-549.

11. Eugênio CS. Avaliação de uma política de visita ampliada sob a ótica dos familiares acompanhantes e equipe assistencial. Porto Alegre [Mestrado em Enfermagem] - Universidade Federal de Ciências da Saúde de Porto Alegre; 2017.

12. Farrell ME, Joseph DH, Schwartz-Barcott D. Visiting hours in the ICU: finding the balance among patient, visitor and staff needs. Nurs Forum 2005; 40(1):18-28.

13. Gibson V, Plowright C, Collins T, Dawson D, Evans S, Gibb P, Lynch F, Mitchell K, Page P, Sturney G. Position statement on visiting in adult critical care units in the UK. Nurs Crit Care 2012; 17(4): 213-2018.

14. Ramos FJ da S, Fumis RRL, Azevedo LCP, Schettino G. Perceptions of an open visitation policy by intensive care unit workers. Ann Intensive Care 2013; 3(1): 34.

15. Athanasiou A, Papathanassoglou ED, Patiraki E, McCarthy MS. Family Visitation in Greek Intensive Care Units: Nurses' Perspective. Am J Crit Care 2014; 23(4): 326-33.

16. Adams S, Herrera A 3rd, Miller L, Soto R. Visitation in the intensive care unit: impact on infection prevention and control. Crit Car Nurs Q 2011; 34(1): 3-10.

17. Giannini A. Open intensive care units: the case in favour. Minerva Anestesiol 2007; 73(5): 299-305.

18. Nassar Junior AP, Besen BAMP, Robinson CC, Falavigna M, Teixeira C, Rosa RG. Flexible Versus Restrictive Visiting Policies in ICUs: A Systematic Review and Meta-Analysis. Crit Care Med 2018; 46(7): 1175-1180. 
19. Rosa RG, Silva DB, Teixeira CT. Projeto UTI Visitas: implementação e avaliação da visita familiar ampliada em UTI's brasileiras. Hospital Moinhos de Vento. Porto Alegre/RS 2017.

20. Rosa RG, Falavigna M, da Silva DB, Sganzerla D, Santos MMS, Kochhann R, et al. Effect of Flexible Family Visitation on Delirium Among Patients in the Intensive Care Unit. JAMA 2019; 322(3): 216-228.

21. Souza RP. Rotinas de humanização em medicina intensiva. São Paulo: Atheneu, 2010.

22. Projeto UTI Visitas [homepage na internet]. A importância do familiar na UTI. 2019. [acesso em 10 de novembro de 2019]. Disponível em < http://www.utivisitas.com.br/ $>$.

23. Ministério da Saúde. Secretaria de Atenção à Saúde. Política Nacional de Humanização. Cadernos Humaniza SUS: formação e intervenção. Brasília: MS, 2010 (MS. Textos Básicos de Saúde).

24. Chapman DK, Mitchell LA, Wright ES, Hopkins RO, Butler JM et al. Satisfaction with elimation of all visitation restrictions in a mixed-profile intensive care unit. Am J Crit Care 2016; 25(1): 46-50.

25. Fumis RR, Ranzani OT, Faria PP, Schettino G. Anxiety, depression, and satisfaction in close relatives of patients in an open visiting policy intensive care unit in Brazil. J Crit Care 2015; 30(2): 440.e1-6.

26. UFSC. Universidade Federal de Santa Catarina [homepage na internet]. Hospital Universitário avalia resultados de projeto para reduzir infecções na UTI. 2018. [acesso em 25 de novembro de 2019]. Disponível em < https://noticias.ufsc.br/2018/09/hospital-universitario-avalia-resultados-de-projetopara-reduzir-infeccoes-na-uti// >

27. Gianinni A, Miccinesi G, Prandi E, Buzzoni C, Borreani C, ODIN Study Group. Partial liberalization of visiting policies and ICU staff: a before-and-after study. Intensive Care Med 2013; 39(12): 21802187.

28. Brasil. Lei $\mathrm{n}^{\circ}$ 8.069, de 13 de julho de 1990. Dispõe sobre o Estatuto da Criança e do Adolescente e dá outras providências. Diário Oficial da União 16 jul 1990.

29. Brasil. Lei no 13.146, de 06 de julho de 2015. Institui a Lei Brasileira de Inclusão da Pessoa com Deficiência (Estatuto da Pessoa com Deficiência). Diário Oficial da União 6 jul 2015.

30. Hospital Universitário Professor Polydoro Ernani de São Thiago. Superintendência. Portaria normativa $\mathrm{n}^{\circ}$ 001, de 24 de maio de 2019. Dispões sobre as normas da visita ampliada na UTI Adulto. Boletim de Serviço HU/UFSC/EBSERH n 17, de 27 de maio de 2019.

31. Reis LCC, Gabarra LM, Moré CLOO. As repercussões do processo de internação em UTI adulto na perspectiva de familiares. Temas psicol 2016; 24(3): 815-828.

32. Rolland, JS. Doença crônica e o ciclo de vida familiar. In: Carter B, Mcgoldrick M. As mudanças no ciclo de vida familiar. 2 ed. Porto Alegre: Artmed; 2001, p. 373-392.

33. Day A, Haj-Bakri S, Lubchanky S, Mehta S. Sleep, anxiety and fatigue in Family members of patientes admitted to the intesive care unit: a questionnaire study. Crit Care 2013; 17(3): R91. 